\title{
Communication
}

\section{The Mutational Concordance of Fixed Formalin Paraffin Embedded and Fresh Frozen Gastro-Oesophageal Tumours Using Whole Exome Sequencing}

\author{
Irene Y. Chong 1,2,*, Naureen Starling ${ }^{2} \mathbb{D}$, Alistair Rust ${ }^{3}$, John Alexander ${ }^{4}$, Lauren Aronson ${ }^{1}$, \\ Marta Llorca-Cardenosa ${ }^{1}$, Ritika Chauhan ${ }^{3}{ }^{-}$, Asif Chaudry ${ }^{2}$, Sacheen Kumar ${ }^{2}{ }^{\mathbb{D}}$, Kerry Fenwick ${ }^{3}$, \\ Ioannis Assiotis ${ }^{3}$, Nik Matthews ${ }^{3}$, Ruwaida Begum ${ }^{2}$, Andrew Wotherspoon ${ }^{2}$, Monica Terlizzo ${ }^{2}$, David Watkins ${ }^{2}$, \\ Ian Chau $\left.{ }^{2}{ }^{\circ},{\text { Christopher J. } \text { Lord }^{4}{ }^{4} \text {, Syed Haider }}^{4}{ }^{(}\right)$, Sheela Rao ${ }^{2}$ and David Cunningham ${ }^{2}$
}

check for

updates

Citation: Chong, I.Y.; Starling, N.; Rust, A.; Alexander, J.; Aronson, L.; Llorca-Cardenosa, M.; Chauhan, R.; Chaudry, A.; Kumar, S.; Fenwick, K.; et al. The Mutational Concordance of Fixed Formalin Paraffin Embedded and Fresh Frozen Gastro-Oesophageal Tumours Using Whole Exome Sequencing. J. Clin. Med. 2021, 10 215. https://doi.org/10.3390/ jcm10020215

Received: 3 December 2020 Accepted: 4 January 2021 Published: 9 January 2021

Publisher's Note: MDPI stays neutral with regard to jurisdictional clai$\mathrm{ms}$ in published maps and institutional affiliations.

Copyright: $(\odot 2021$ by the authors. Licensee MDPI, Basel, Switzerland. This article is an open access article distributed under the terms and conditions of the Creative Commons Attribution (CC BY) license (https:// creativecommons.org/licenses/by/ $4.0 /)$.
1 The Division of Molecular Pathology, The Institute of Cancer Research, 237 Fulham Road, London SW3 6JB, UK; lauren.aronson@icr.ac.uk (L.A.); marta.llorca@icr.ac.uk (M.L.-C.)

2 The Royal Marsden Hospital NHS Foundation Trust, 203 Fulham Road, London SW3 6JJ, UK; Naureen.starling@rmh.nhs.uk (N.S.); asif.chaudry@rmh.nhs.uk (A.C.); sacheen.kumar@rmh.nhs.uk (S.K.); ruwaida.begum@rmh.nhs.uk (R.B.); andrew.wotherspoon@rmh.nhs.uk (A.W.); monica.terlizzo@rmh.nhs.uk (M.T.); david.watkins@rmh.nhs.uk (D.W.); ian.chau@rmh.nhs.uk (I.C.); sheela.rao@rmh.nhs.uk (S.R.); david.cunningham@rmh.nhs.uk (D.C.)

3 The Tissue Profiling Unit, The Institute of Cancer Research, 237 Fulham Road, London SW3 6JB, UK; alistair.rust@icr.ac.uk (A.R.); ritika.chauhan@icr.ac.uk (R.C.); kerry.fenwick@icr.ac.uk (K.F.); ioannis.assiotis@icr.ac.uk (I.A.); nik.matthews@icr.ac.uk (N.M.)

4 Breast Cancer Now Toby Robins Research Centre, The Institute of Cancer Research, London SW3 6JB, UK John.Alexander@icr.ac.uk (J.A.); Chris.lord@icr.ac.uk (C.J.L.); Syed.Haider@icr.ac.uk (S.H.)

* Correspondence: irene.chong@icr.ac.uk; Tel.: +44-0207-153-5138

Abstract: 1. Background: The application of massively parallel sequencing has led to the identification of aberrant druggable pathways and somatic mutations within therapeutically relevant genes in gastro-oesophageal cancer. Given the widespread use of formalin-fixed paraffin-embedded (FFPE) samples in the study of this disease, it would be beneficial, especially for the purposes of biomarker evaluation, to assess the concordance between comprehensive exome-wide sequencing data from archival FFPE samples originating from a prospective clinical study and those derived from fresh-frozen material. 2. Methods: We analysed whole-exome sequencing data to define the mutational concordance of 16 matched fresh-frozen and FFPE gastro-oesophageal tumours $(N=32)$ from a prospective clinical study. We assessed DNA integrity prior to sequencing and then identified coding mutations in genes that have previously been implicated in other cancers. In addition, we calculated the mutant-allele heterogeneity (MATH) for these samples. 3. Results: Although there was increased degradation of DNA in FFPE samples compared with frozen samples, sequencing data from only two FFPE samples failed to reach an adequate mapping quality threshold. Using a filtering threshold of mutant read counts of at least ten and a minimum of $5 \%$ variant allele frequency (VAF) we found that there was a high median mutational concordance of $97 \%$ (range $80.1-98.68 \%$ ) between fresh-frozen and FFPE gastro-oesophageal tumour-derived exomes. However, the majority of FFPE tumours had higher mutant-allele heterogeneity (MATH) scores when compared with corresponding frozen tumours $(p<0.001)$, suggesting that FFPE-based exome sequencing is likely to over-represent tumour heterogeneity in FFPE samples compared to fresh-frozen samples. Furthermore, we identified coding mutations in 120 cancer-related genes, including those associated with chromatin remodelling and Wnt/ $\beta$-catenin and Receptor Tyrosine Kinase signalling. 4. Conclusions: These data suggest that comprehensive genomic data can be generated from exome sequencing of selected DNA samples extracted from archival FFPE gastro-oesophageal tumour tissues within the context of prospective clinical trials.

Keywords: gastro-oesophageal cancer; mutational concordance; exome sequencing; formalin fixed paraffin embedded; biomarkers 


\section{Introduction}

Gastric and oesophageal cancers are, respectively, the third and seventh leading causes of cancer-related deaths [1-3]. Disease relapse following first-line treatment in patients with advanced disease is frequent, with limited subsequent treatment options. Previously studied targeted therapies in patients with advanced gastro-oesophageal cancer include inhibitors of erythroblastic oncogene B (ERBB2) [4], epidermal growth factor receptor (EGFR) [5,6], vascular endothelial growth factor (VEGF) [7], vascular endothelial growth factor receptor (VEGFR2) [8,9], and poly (ADP-ribose) polymerase (PARP) [10]. However, an improved understanding of individual patient responses is required to identify actionable mechanisms of treatment response and resistance. Genome-wide DNA sequencing studies have confirmed that gastro-oesophageal adenocarcinomas are highly mutated and heterogeneous tumours [11,12]. We and others have identified aberrant druggable pathways and somatic mutations within therapeutically relevant genes in the treatment of naïve frozen gastro-oesophageal tumours using massively parallel sequencing techniques [11,13-15]. For the purposes of biomarker evaluation, it would be beneficial to utilise whole-exome DNA sequencing to generate comprehensive genomic data that could be compared with clinical response and outcome within mature phase III studies. Unfortunately, only formalin-fixed paraffin-embedded (FFPE) tissues are available for genomic evaluation in most of these trials; this could potentially be problematic as the process of tissue immobilisation by the FFPE process can result in cross-linked and fragmented DNA that may not be fit for purpose for massively parallel sequencing [16]. It is, therefore, important to understand the level of mutational concordance between frozen and FFPE tumours to assess the utility of next-generation sequencing of DNA extracted from FFPE tissues. Here, we describe an analysis of whole-exome sequencing data to define the mutational concordance of DNA extracted from matched fresh frozen and FFPE gastro-oesophageal tumours, and to estimate the feasibility of this approach within the context of prospective clinical trials.

\section{Experimental Section}

\subsection{Sample Description and Preparation}

Snap frozen and matched FFPE gastro-oesophageal tumour biopsies used for exome sequencing were obtained from patients at the time of endoscopic ultrasound staging, prior to treatment by the same endoscopist at the Royal Marsden Hospital, UK. The biopsies were fixed in neutral buffered formalin for 5-8 h. Oesophageal tumour samples with malignant cell purities of over $70 \%$ were selected for DNA extraction and subsequent whole-exome sequencing. Signed written informed consent from each patient was obtained before recruitment to the study according to regulations of the local ethics review board.

\subsection{Genomic DNA Extraction and Whole-Exome}

Genomic DNA was isolated from tumour biopsies using the DNeasy Blood and Tissue kit (Qiagen, Hilden, Germany) and quantified using Qubit fluorometric quantitation (Invitrogen Life Technologies, Carlsbad, CA, USA). Genomic DNA was fragmented to 200 basepairs (bps) using a Covaris E Series instrument (Covaris Inc., Woburn, MA, USA). The resultant library was subjected to DNA capture using the $50 \mathrm{Mb}$ SureSelect Human All Exon V5 kit (Agilent, Santa Clara, CA, USA). DNA capture was carried out, and Illumina paired-end libraries were prepared from the captured target regions and quantified using a Bioanalyzer DNA chip (Agilent). This process was then followed by sequencing on a HiSeq2500 platform (Illumina, San Diego, CA, USA), acquiring $2 \times 100$ bps reads. Bcl2fastq software (v1.8.4, Illumina) was used for converting the raw basecalls to fastqs and to further demultiplex the sequencing data. The demultiplexed paired-end fastq files were used for further analysis. 


\subsection{Read Mapping and Detection of Mutations from Exome Sequencing}

BWA-mem (v0.7.5a) was used to align reads to the human reference genome (GRCh37) [17]. Variant calling was carried out using the Broad Best Practice pipeline with standard settings [18]. In summary, GATK (v3.3-0) was used to detect frameshifts and MuTect (v1.1.4) was used to detect point mutations. The effects of single-point mutations were determined by SnpEff (v3.3h). Candidate mutations were selected using the following list of heuristic rules: (1) variants detected at a mutant allele frequency (MAF) of greater than $5 \%$ in any of the 1000 Genomes project populations were excluded from analysis, (2) variants called in regions not covered by the exome capture probes were excluded, (3) variants marked as low quality (QUAL below 30) were excluded, and (4) variants not reaching a depth threshold of 10 reads were excluded.

\section{Results}

\subsection{Clinicopathological Features of Patients}

All patients were treatment-naïve at the time of biopsy retrieval. The median age was 64 years for the 16 patients included in this study (Table 1). The majority were male (81.2\%). The most common disease site was at the gastro-oesophageal junction (GOJ, 68.8\%). The GOJ and gastric tumours were adenocarcinomas (93.8\%) that were either moderately or poorly differentiated (grade 2 or 3). The remaining cancer was an early, well-differentiated (grade 1) neuroendocrine tumour located in the distal oeosphagus. The majority of tumours were locally advanced (T3 N0/1 M0, 62\%). Four patients had early disease (T1/2 N0, M0, $25 \%$ ), and two patients presented with metastatic disease (T3 N1 M1, 12.5\%). The storage period of the tissues ranged from 4 to 10 years, with a median time of 8.5 years.

Table 1. Clinicopathological characteristics of patients.

\begin{tabular}{|c|c|}
\hline Characteristic & $(N=16)$ \\
\hline \multicolumn{2}{|c|}{ Age at diagnosis } \\
\hline Median-y & 64 \\
\hline Range-y & $22-82$ \\
\hline \multicolumn{2}{|c|}{ Sex-No. $(\%)$} \\
\hline Male & $13(81.2)$ \\
\hline Female & $3(18.8)$ \\
\hline \multicolumn{2}{|c|}{ Site of tumour-No. (\%) } \\
\hline Distal oesophagus & $1(6.3)$ \\
\hline GOJ type I & $3(18.7)$ \\
\hline GOJ type II & $4(25)$ \\
\hline GOJ type III & $4(25)$ \\
\hline Stomach & $4(25)$ \\
\hline \multicolumn{2}{|c|}{ Histology-No. (\%) } \\
\hline Adenocarcinoma & $15(93.8)$ \\
\hline Neuroendocrine & $1(6.2)$ \\
\hline \multicolumn{2}{|c|}{ Grade-No. (\%) } \\
\hline 1 & $1(6.2)$ \\
\hline 2 & $5(31.3)$ \\
\hline 3 & $10(62.5)$ \\
\hline \multicolumn{2}{|c|}{ TNM Stage-No. (\%) } \\
\hline T1/2 N0 M0 & $4(25)$ \\
\hline T3 N0/1 M0 & $10(62.5)$ \\
\hline T3 N1 M1 & $2(12.5)$ \\
\hline \multicolumn{2}{|c|}{ Time from biopsy to sequencing } \\
\hline Median-y & 8.5 \\
\hline Range-y & $4-10$ \\
\hline
\end{tabular}




\subsection{Assessment of DNA Integrity}

We observed that there was a significant difference in the concentration of doublestranded DNA extracted from frozen compared with FFPE oesophageal tumour $(p=0.0026$, Mann-Whitney U test), suggesting improved integrity of DNA extracted from frozen samples and increased degradation of FFPE biospecimens (Figure 1A) [19]. However, there was no significant difference in either the total quantity of pre-hybridisation PCR product generated or the number of PCR cycles required to generate the pre-hybridisation library prior to exome sequencing (Figure 1B,C). Following exome sequencing, mutation filtering was applied including mapping quality threshold of $\geq 30$, depth threshold of $\geq 10$ reads, and variant allele frequency (VAF) threshold of $\geq 0.05$. Of note, in the absence of matched blood samples, many germline variants are likely to exist in our mutational repertoire. By applying these thresholds, mutation calls detected in frozen tumour samples were considered a gold standard, allowing for the calculation of true positive, false positive, and false negative rates. For each set of thresholds, combined numbers for sensitivity, precision/positive predictive value (PPV), and F-score were calculated (Table 2). The two sets of thresholds with the highest PPV and F-scores were for mutant read counts of ten or more and a minimum of $5 \%$ VAF. We observed that all of the 16 frozen samples achieved adequate exome coverage and depth. However, two of the 16 FFPE samples (samples 178 and 260) did not achieve the minimum median depth threshold of $50 \times$. The ages of the two FFPE specimens that failed were 5 years and 10 years, respectively (the range for this cohort was 4-10 years). Whilst the initial starting quantities of DNA and following fragmentation were adequate, the total amount of post-adapter-ligation DNA was lower than expected (less than $400 \mathrm{ng}$ ), indicating inferior DNA quality. These samples failed the quality control criteria and were excluded from further analyses.

Table 2. Sensitivity, precision/positive predictive value (PPV), and F-Score for selected variant allele frequency (VAF) and tumour depth thresholds.

\begin{tabular}{|c|c|c|c|c|}
\hline VAF (\%) & Tumour Depth (X) & Combined Sensitivity & Combined Precision PPV & Combined F Score \\
\hline 2 & 5 & 0.775700935 & 0.83 & 0.801932367 \\
\hline 5 & 5 & 0.775700935 & 0.83 & 0.801932367 \\
\hline 10 & 5 & 0.76076555 & 0.81122449 & 0.785185185 \\
\hline 15 & 5 & 0.712643678 & 0.765432099 & 0.738095238 \\
\hline 20 & 5 & 0.732283465 & 0.801724138 & 0.765432099 \\
\hline 2 & 10 & 0.778301887 & 0.829145729 & 0.802919708 \\
\hline 5 & 10 & 0.778301887 & 0.829145729 & 0.802919708 \\
\hline 10 & 10 & 0.763285024 & 0.81025641 & 0.786069652 \\
\hline 15 & 10 & 0.715116279 & 0.763975155 & 0.738738739 \\
\hline 20 & 10 & 0.744 & 0.801724138 & 0.771784232 \\
\hline 2 & 15 & 0.773584906 & 0.83248731 & 0.80195599 \\
\hline 5 & 15 & 0.773584906 & 0.83248731 & 0.80195599 \\
\hline 10 & 15 & 0.758454106 & 0.813471503 & 0.785 \\
\hline 15 & 15 & 0.709302326 & 0.767295597 & 0.737160121 \\
\hline 20 & 15 & 0.736 & 0.807017544 & 0.769874477 \\
\hline 2 & 20 & 0.763033175 & 0.829896907 & 0.795061728 \\
\hline 5 & 20 & 0.763033175 & 0.829896907 & 0.795061728 \\
\hline 10 & 20 & 0.747572815533981 & 0.810526316 & 0.777777778 \\
\hline 15 & 20 & 0.695906433 & 0.762820513 & 0.727828746 \\
\hline 20 & 20 & 0.717741935 & 0.801801802 & 0.757446809 \\
\hline 2 & 25 & 0.759615385 & 0.822916667 & 0.79 \\
\hline 5 & 25 & 0.759615385 & 0.822916667 & 0.79 \\
\hline 10 & 25 & 0.748768473 & 0.808510638 & 0.777493606 \\
\hline 15 & 25 & 0.704142012 & 0.767741935 & 0.734567901 \\
\hline 20 & 25 & 0.729508197 & 0.809090909 & 0.767241379 \\
\hline 2 & 30 & 0.747572816 & 0.814814815 & 0.779746835 \\
\hline 5 & 30 & 0.747572816 & 0.814814815 & 0.779746835 \\
\hline 10 & 30 & 0.736318408 & 0.8 & 0.766839378 \\
\hline 15 & 30 & 0.694610778 & 0.753246753 & 0.722741433 \\
\hline 20 & 30 & 0.716666667 & 0.788990826 & 0.751091703 \\
\hline
\end{tabular}




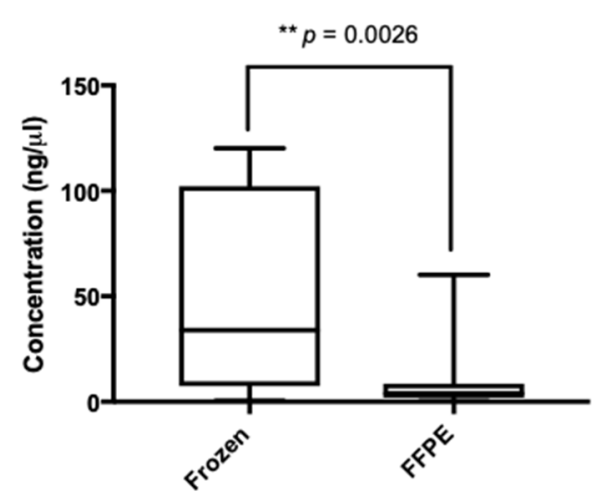

(A)

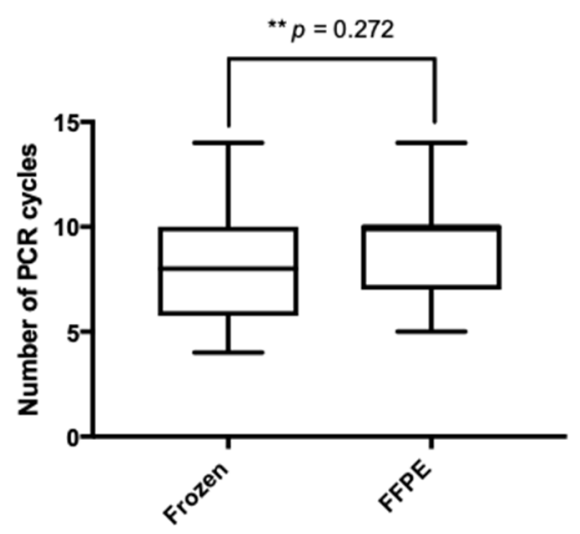

(C)

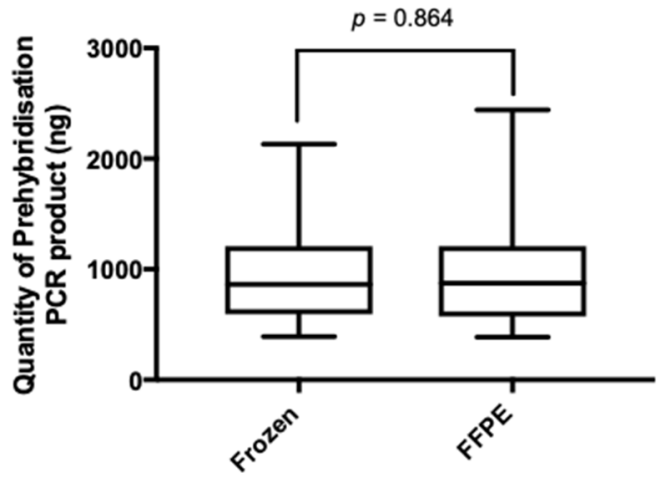

(B)

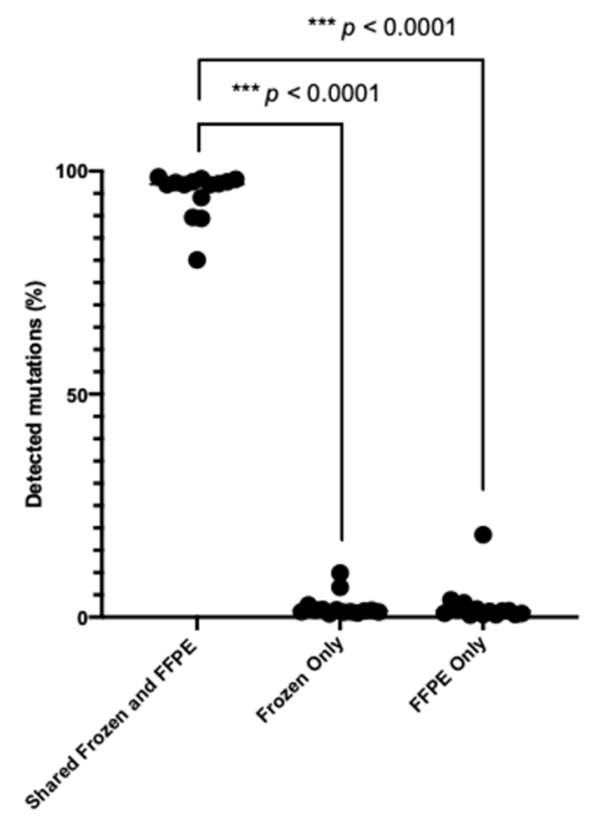

(D)

Figure 1. Mutational concordance between frozen and formalin-fixed paraffin-embedded (FFPE) gastro-oesophageal tumour samples. Box and whiskers plots showing the distribution of (A). The concentration of double-stranded DNA (nanograms per microlitre) extracted from FFPE and frozen gastro-oesophageal tumours. An increased double-stranded DNA yield was extracted from frozen tumour tissues compared with FFPE tissue $(p=0.0026$, $)($ B). Prehybridisation PCR product (nanograms) (C). The number of PCR cycles required to generate the pre-hybridisation library from FFPE and frozen gastro-oesophageal tumour samples. No difference between FFPE and frozen samples was observed in terms of the overall quantity of pre-hybridisation PCR product generated, nor in terms of the number of PCR cycles required to generate the pre-hybridisation library prior to sequencing (D). Bar graph showing a high mutational concordance (range $80.1 \%$ to $98.68 \%$ ) in terms of the percentage of shared mutations detected (in both frozen and FFPE samples) compared with mutations unique to frozen samples and FFPE samples.

\subsection{Mutational Concordance between Frozen and FFPE Oesophageal Tumour Samples}

To assess the mutational concordance between matched frozen and FFPE-derived gastro-oesophageal tumour DNA in the 14 matched samples that passed quality control criteria $(N=28)$, we cross-referenced mutations detected from exome sequencing. We observed that there was a high median mutational concordance of $97.07 \%$ (range $80.1 \%$ to $98.68 \%$ ) between fresh-frozen and FFPE gastro-oesophageal tumour samples (Figure 1D, Table 3). There was no difference overall in the percentage of unique mutations found in DNA derived from FFPE compared with frozen tumour tissue $(p=0.41$, Mann-Whitney U test). Given that $93 \%(90 / 96)$ of randomly selected mutations have previously been 
validated with Sanger sequencing, and that $95 \%(1791 / 1883)$ of mutations were recognised by both exome sequencing and the Ion Proton platform from our previous study [14], our current results demonstrate the feasibility of exome sequencing of FFPE-derived DNA samples from gastro-oesophageal tumours that have passed the described quality control criteria.

Table 3. The of percentage mutational concordance of matched fresh frozen and formalin-fixed paraffin-embedded (FFPE) gastro-oesophageal tumour samples.

\begin{tabular}{|c|c|c|c|}
\hline Patient & Sample & Mutations Unique to Sample & $\%$ Unique to Sample \\
\hline \multirow{3}{*}{169} & Frozen & 1955 & 6.72 \\
\hline & FFPE & 1129 & 3.88 \\
\hline & Shared & 25,997 & 89.40 \\
\hline \multirow{3}{*}{170} & Frozen & 456 & 1.65 \\
\hline & FFPE & 250 & 0.90 \\
\hline & Shared & 26,958 & 97.45 \\
\hline \multirow{3}{*}{172} & Frozen & 471 & 1.73 \\
\hline & FFPE & 361 & 1.32 \\
\hline & Shared & 26,424 & 96.95 \\
\hline \multirow{3}{*}{176} & Frozen & 853 & 2.72 \\
\hline & FFPE & 1017 & 3.24 \\
\hline & Shared & 29,508 & 94.04 \\
\hline \multirow{3}{*}{177} & Frozen & 476 & 1.42 \\
\hline & FFPE & 6205 & 18.48 \\
\hline & Shared & 26,896 & 80.10 \\
\hline \multirow{3}{*}{187} & Frozen & 3056 & 9.91 \\
\hline & FFPE & 146 & 0.47 \\
\hline & Shared & 27,630 & 89.61 \\
\hline \multirow{3}{*}{195} & Frozen & 204 & 0.72 \\
\hline & FFPE & 168 & 0.60 \\
\hline & Shared & 27,790 & 98.68 \\
\hline \multirow{3}{*}{203} & Frozen & 428 & 1.56 \\
\hline & FFPE & 233 & 0.85 \\
\hline & Shared & 26,811 & 97.59 \\
\hline \multirow{3}{*}{218} & Frozen & 311 & 1.14 \\
\hline & FFPE & 154 & 0.57 \\
\hline & Shared & 26,738 & 98.29 \\
\hline \multirow{3}{*}{220} & Frozen & 317 & 1.18 \\
\hline & FFPE & 493 & 1.84 \\
\hline & Shared & 25,978 & 96.98 \\
\hline \multirow{3}{*}{249} & Frozen & 257 & 0.92 \\
\hline & FFPE & 408 & 1.47 \\
\hline & Shared & 27,119 & 97.61 \\
\hline \multirow{3}{*}{254} & Frozen & 327 & 1.23 \\
\hline & FFPE & 159 & 0.60 \\
\hline & Shared & 26,076 & 98.17 \\
\hline \multirow{3}{*}{259} & Frozen & 325 & 1.20 \\
\hline & FFPE & 441 & 1.63 \\
\hline & Shared & 26,234 & 97.16 \\
\hline \multirow{3}{*}{267} & Frozen & 468 & 1.61 \\
\hline & FFPE & 413 & 1.42 \\
\hline & Shared & 28,209 & 96.97 \\
\hline
\end{tabular}




\subsection{Detection of Mutations within Cancer-Related Genes}

To identify coding mutations in genes from exome sequencing that have also been implicated in other cancers, we correlated genes harbouring frameshift, non-synonymous, splice site, and stop-gained mutations with genes in the Cancer Genome Census (CGC) [20]. Overall, this comparison identified 120 cancer-related genes in the gastro-oesophageal samples from this study, with an average of 12 potentially deleterious CGC mutations (range 750) present in each sample (Figure 2). These mutations were further analysed to determine the dysregulation of cancer-associated pathways. Using this approach, we observed coding mutations in tumour-suppressor genes usually required for normal chromatin remodelling, including ARID1A (AT-rich interaction domain 1A gene), BRD3 (Bromodomain-containing protein 3 gene), and SMARCA4 (SWI/SNF-Related Matrix-Associated Actin-Dependent Regulator of Chromatin Subfamily A, Member 4 gene). In addition, 8 out of 16 tumours harboured mutations in well-established DNA repair-related tumour-suppressor genes, including FANCE (Fanconi Anemia Complementation Group E gene), FANCF (Fanconi Anaemia Complementation Group F gene), MSH6 (MutS Homolog 6 gene), PMS1 (PMS1 Homolog1, Mismatch Repair System Component gene), PMS2 (PMS1 Homolog 2, Mismatch Repair System Component gene), ERCC2 (ERCC Excision Repair 2, TFIIH Core Complex Helicase Subunit gene), or SETD2 (SET Domain Containing 2 gene), suggestive of disrupted DNA repair pathway signalling in these tumours. Coding mutations in genes involved in Wnt signalling were detected, including mutations in BCL9 (B-Cell CLL/Lymphoma 9 gene) and AXIN1 (Axin 1 gene). Coding mutations in TP53 were detected in 4 out of 16 tumours from exome sequencing. We also identified mutations in genes involved in RAS/RAF signalling, including KRAS (Kirsten ras oncogene) and BRAF (B-Raf Proto-Oncogene, Serine/Threonine Kinase gene). Mutations in therapeutically relevant genes were also observed, including those in MET (MET Proto-Oncogene, Receptor Tyrosine Kinase gene) and FGFR1 (Fibroblast Growth Factor Receptor 1 gene).

\subsection{Intratumoural Genetic Heterogeneity}

Gastro-oesophageal tumours are known to be heterogeneous cancers [14,21]. Intratumoural heterogeneity with respect to actionable mutations has clinical implications for how targeted therapies might work [22,23]. Genomically distinct subpopulations of cells lead to differences among mutated loci in terms of the fraction of sequence reads displaying a mutant allele. A heterogeneous tumour will likely have a wider distribution of mutantallele fractions among loci centred at a lower fraction, compared with a homogeneous tumour [24]. Taking this into consideration, we analysed exome sequencing results for each of the frozen and FFPE tumours. Moreover, we calculated the mutant-allele heterogeneity (MATH) score as the ratio of the width to the centre of its distribution of mutant-allele fractions among tumour-specific mutated loci (Supplementary Figure S1). We observed that the median MATH score for the frozen tumours was 32.95 (range 17.4 to 96.6), indicating notable differences in inter-tumoural heterogeneity in this set of gastro-oesophageal samples. The majority of the FFPE tumours (11 out of 14 samples) had higher MATH scores when compared with the corresponding frozen tumours $(p<0.001$ Wilcoxon rank test, Figure $3 \mathrm{~A}$ ), suggesting that this analysis is likely to over-represent tumour heterogeneity in FFPE samples. In addition, the number of clonal clusters calculated by MATH was discordant in 9 out of 14 matched samples (Figure 3B). Although the median mutational concordance between fresh-frozen and FFPE gastro-oesophageal tumour samples was high (median 97\%, range 80.1-98.68\%), MATH analysis to assess tumour heterogeneity was not found to be reliable in FFPE samples. 


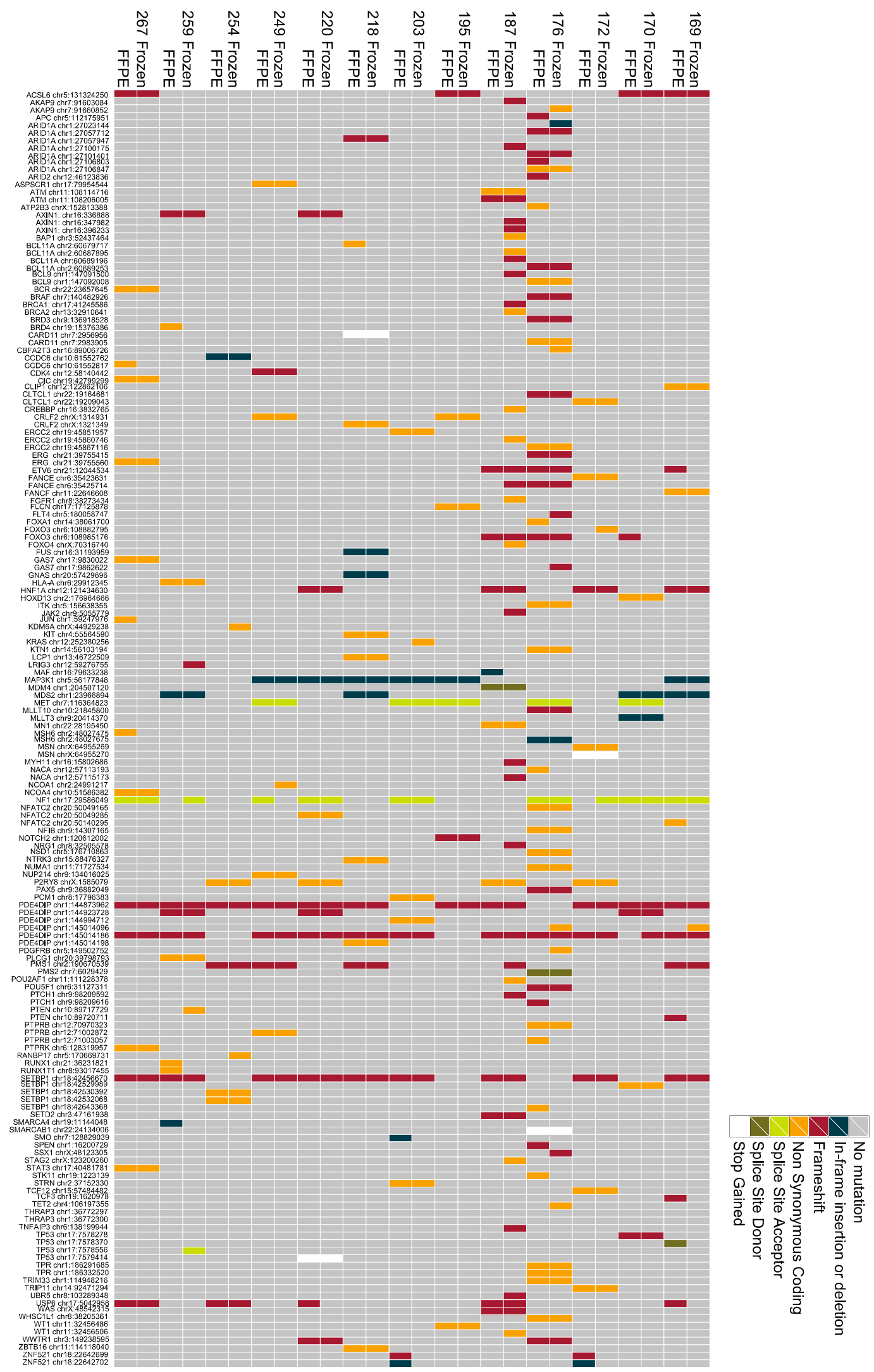

Figure 2. Detection of cancer-related genes from exome sequencing of gastro-oesophageal tumour DNA. Commutation plot showing the presence of mutations in genes from the Cancer Genome Census from exome sequencing of DNA extracted from frozen and formalin-fixed paraffin-embedded (FFPE) oesophageal tumours. 

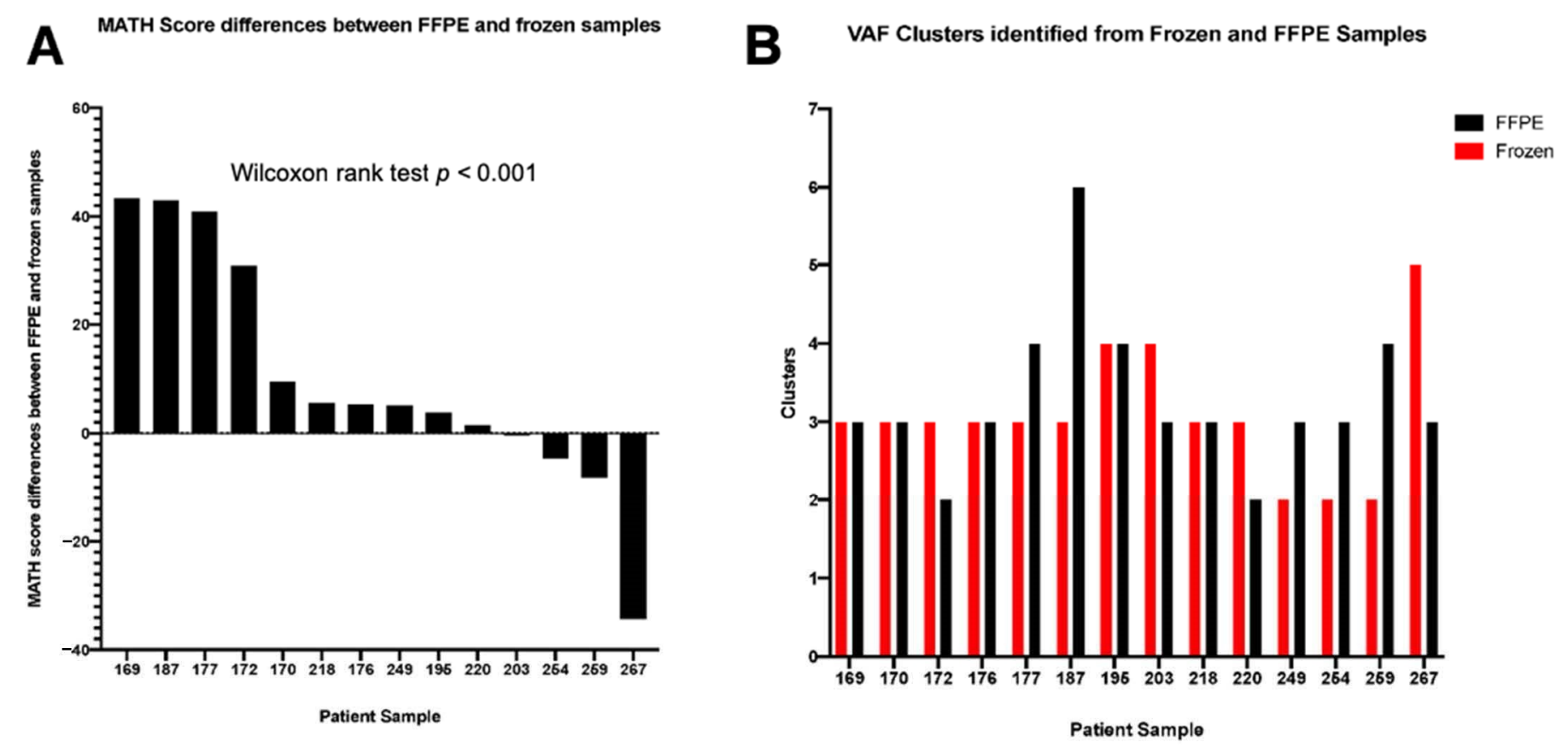

Figure 3. Differences in mutant-allele heterogeneity (MATH) scores and variant allele frequency (VAF) clusters identified from matched formalin-fixed paraffin-embedded (FFPE) and frozen gastro-oesophageal tumours. Histogram illustrating (A) differences in MATH scores between FFPE and frozen tumour samples-11 out of 14 FFPE tumours had higher MATH scores when compared with the corresponding frozen tumours ( $p<0.001$ Wilcoxon rank test); and (B) VAF clusters identified from FFPE and frozen samples.

\section{Discussion}

Considering the potential clinical impact of dissecting molecular mechanisms of treatment response and resistance within prospective clinical trials where only FFPE samples are available for analysis [25], the main purpose of this study was to assess the feasibility of using DNA extracted from FFPE gastro-oesophageal tumour for massively parallel sequencing. It is acknowledged that DNA cross-linking, degradation, and fragmentation occurring during the FFPE process has the potential to influence the reliability of mutational sequencing data [26-29]. Taking matched FFPE and frozen melanoma specimens as examples, a comparison of whole-exome sequencing data from 10 tumours revealed a very low overall mutational concordance (average $43.2 \%$ ). However, the most clinically actionable mutations for this tumour type (BRAF and NRAS) were found to be concordant [30]. The authors from this study concluded that specialised library construction to account for low quality DNA is necessary before this approach could be used for routine clinical decision making. In contrast, studies relating to other tumour types and utilizing different massively parallel sequencing techniques have yielded more promising results; the concordance rate was found to be up to $96.8 \%$ in a lung cancer study comparing the variants of 27 cancer-related genes in 16 matched FFPE and frozen samples [31]. Mutational comparisons have also been undertaken in colorectal cancer (CRC) specimens; the detected concordance rate was up to $81.9 \%$ in a study of 33 matched metastatic CRC samples [32]. In a cohort of 10 paired metastatic liver CRC specimens, a high mutational concordance was observed when 212 amplicon regions in 48 cancer-related genes were sequenced, revealing 21 identical mutation calls and only two differing mutations [33]. Furthermore, Gao et al. conducted an extensive study using a 22-gene panel detecting 103 hotspot mutations in paired FFPE and fresh-frozen primary CRC tissues from 118 patients [34]. The investigators identified a concordance rate ranging from $73.8 \%$ to $100 \%$ and highlighted that important differences exist between the two tissue types.

We approached this problem by assessing DNA integrity prior to sequencing and analysing whole-exome sequencing data to define the mutational concordance of matched 
fresh-frozen and FFPE gastro-oesophageal tumours. As expected, DNA degradation was more pronounced in the FFPE biospecimens compared with the matched frozen samples. However, there was no significant differences in either the total quantity of pre-hybridisation PCR product generated or the number of PCR cycles required to generate the pre-hybridisation library prior to exome sequencing. Only two out of 16 FFPE samples failed quality control criteria with the inability to achieve the minimum median depth threshold of $50 \times$. In the absence of normal/germline samples, we considered all variants likely to include many germline variants. Based on these variants, the subsequent calculation of PPV and F-scores, allowing for the calculation of true-positive, false-positive, and false-negative rates, using frozen tumour samples as a gold standard, identified the optimal filtering threshold as mutant read counts of 10 or more and a minimum of $5 \%$ VAF. Using this threshold, we observed a high median mutational concordance of $97 \%$ between DNA derived from fresh frozen and FFPE gastro-oesophageal tumours. Consistent with the literature, we also identified frequent mutations in genes responsible for chromatin remodelling, Wnt/ $\beta$-catenin, and Receptor Tyrosine Kinase signalling [13]. Finally, we assessed intratumoural heterogeneity by calculating the MATH score, and the ratio of the width to the centre of its distribution of mutant-allele fractions among tumour-specific mutated loci, for each sample. We found that most FFPE gastro-oesophageal tumours in this study had higher MATH scores compared with the corresponding frozen tumours. FFPE samples are likely to over-estimate tumour heterogeneity due to the presence of artefactual substitutions in FFPE samples [35]. This result may lead to a more significant variation in observed VAFs, resulting in a higher MATH score.

Focusing on the two FFPE specimens that failed sequencing quality control, we have scrutinised the clinicopathological characteristics of patients included in this study, as well as the raw data generated after DNA extraction and before massively parallel sequencing, to evaluate whether, at any stage, sequencing failure could have been predicted. We found that none of the clinical characteristics were responsible. In particular, the age of the two FFPE specimens that failed were five years and ten years, respectively (the range for this cohort was 4-10 years). Furthermore, we confirmed that the initial quantities of DNA and following fragmentation were indeed adequate. However, the total amount of postadapter-ligation DNA was lower than expected (less than $400 \mathrm{ng}$ ), which is an indication of inferior DNA quality. Whilst this finding could serve as a warning for investigators, we cannot definitively conclude that this factor alone should preclude the commencement of exome sequencing in future studies.

Our findings support the validity of massively parallel sequencing of FFPE gastrooesophageal tissues as a discovery tool, recognising that only archival tumour blocks are available in the majority of completed phase III studies. Through rigorous assessment of DNA integrity and application of an optimal filtering threshold, a high level of mutational concordance between FFPE and frozen tissues can be achieved. However, subsequent orthogonal validation of actionable mutations is of utmost importance. In contrast, the assessment of intratumoural heterogeneity using the distribution of mutant allele fractions in FFPE gastro-oesophageal samples is much less reliable.

Supplementary Materials: The following are available online at https:/ / www.mdpi.com/2077-038 3/10/2/215/s1, Figure S1: MATH Profiles of intra-tumoural heterogeneity in matched grozen and FFPE gastro-oesophageal tumours.

Author Contributions: Conceptualization, I.Y.C., D.C., N.S., D.W., I.C., and S.R.; methodology, I.Y.C., L.A., A.C., S.K., K.F., I.A., N.M., A.W., and M.T.; software, S.H., J.A., A.R., and R.C.; validation, L.A. and M.L.-C.; formal analysis, I.Y.C., A.R., R.C., J.A., and S.H.; data curation, L.A., M.L.-C., R.B.; writing-original draft preparation, I.Y.C.; writing - review and editing, all authors. All authors have read and agreed to the published version of the manuscript. 
Funding: We thank the following for financially supporting this work: the Thornton Foundation and the Royal Marsden Cancer Charity (funding to I.Y.C.), the Medical Research Council (I.Y.C. and D.C.), Cancer Research UK as part of Programme Funding (C.J.L.), Breast Cancer Now, working in partnership with Walk the Walk (S.H.). This work represents independent research supported by the National Institute for Health Research (NIHR) Biomedical Research Centre at The Royal Marsden NHS Foundation Trust and the Institute of Cancer Research, London. The views expressed are those of the authors and not necessarily those of the NIHR or the Department of Health and Social Care.

Institutional Review Board Statement: The study was conducted according to the guidelines of the Declaration of Helsinki, and approved by the Royal Marsden Hospital Ethics (CCR 2110).

Informed Consent Statement: Informed consent was obtained from all subjects involved in the study.

Data Availability Statement: The raw data supporting the conclusions of this article are available on request to the corresponding author.

Conflicts of Interest: The authors declare no conflict of interest.

\section{References}

1. Siegel, R.L.; Miller, K.D.; Jemal, A. Cancer statistics. CA A Cancer J. Clin. 2017, 67, 7-30. [CrossRef] [PubMed]

2. Rustgi, A.K.; El-Serag, H.B. Esophageal Carcinoma. N. Engl. J. Med. 2014, 371, 2499-2509. [CrossRef] [PubMed]

3. Siegel, R.L.; Miller, K.D.; Jemal, A. Cancer statistics. CA Cancer J. Clin. 2019, 69, 7-34. [CrossRef] [PubMed]

4. Bang, Y.-J.; Van Cutsem, E.; Feyereislova, A.; Chung, H.C.; Shen, L.; Sawaki, A.; Lordick, F.; Ohtsu, A.; Omuro, Y.; Satoh, T.; et al. Trastuzumab in combination with chemotherapy versus chemotherapy alone for treatment of HER2-positive advanced gastric or gastro-oesophageal junction cancer (ToGA): A phase 3, open-label, randomised controlled trial. Lancet 2010, 376, 687-697. [CrossRef]

5. Dutton, S.J.; Ferry, D.R.; Blazeby, J.M.; Abbas, H.; Dahle-Smith, A.; Mansoor, W.; Thompson, J.; Harrison, M.; Chatterjee, A.; Falk, S.; et al. Gefitinib for oesophageal cancer progressing after chemotherapy (COG): A phase 3, multicentre, double-blind, placebo-controlled randomised trial. Lancet Oncol. 2014, 15, 894-904. [CrossRef]

6. Waddell, T.; Chau, I.; Cunningham, D.; Gonzalez, D.; Okines, A.F.C.; Wotherspoon, A.; Saffery, C.; Middleton, G.; Wadsley, J.; Ferry, D.; et al. Epirubicin, oxaliplatin, and capecitabine with or without panitumumab for patients with previously untreated advanced oesophagogastric cancer (REAL3): A randomised, open-label phase 3 trial. Lancet Oncol. 2013, 14, 481-489. [CrossRef]

7. Ohtsu, A.; Shah, M.A.; Van Cutsem, E.; Rha, S.Y.; Sawaki, A.; Park, S.R.; Lim, H.Y.; Yamada, Y.; Wu, J.; Langer, B.; et al. Bevacizumab in Combination with Chemotherapy as First-Line Therapy in Advanced Gastric Cancer: A Randomized, DoubleBlind, Placebo-Controlled Phase III Study. J. Clin. Oncol. 2011, 29, 3968-3976. [CrossRef]

8. Fuchs, C.S.; Tomasek, J.; Yong, C.J.; Dumitru, F.; Passalacqua, R.; Goswami, C.; Safran, H.; Dos Santos, L.V.; Aprile, G.; Ferry, D.R.; et al. Ramucirumab monotherapy for previously treated advanced gastric or gastro-oesophageal junction adenocarcinoma (REGARD): An international, randomised, multicentre, placebo-controlled, phase 3 trial. Lancet 2014, 383, 31-39. [CrossRef]

9. Wilke, H.; Muro, K.; Van Cutsem, E.; Oh, S.-C.; Bodoky, G.; Shimada, Y.; Hironaka, S.; Sugimoto, N.; Lipatov, O.; Kim, T.-Y.; et al. Ramucirumab plus paclitaxel versus placebo plus paclitaxel in patients with previously treated advanced gastric or gastro-oesophageal junction adenocarcinoma (RAINBOW): A double-blind, randomised phase 3 trial. Lancet Oncol. 2014, 15, 1224-1235. [CrossRef]

10. Bang, Y.-J.; Xu, R.-H.; Chin, K.; Lee, K.-W.; Park, S.H.; Rha, S.Y.; Shen, L.; Qin, S.; Xu, N.; Im, S.-A.; et al. Olaparib in combination with paclitaxel in patients with advanced gastric cancer who have progressed following first-line therapy (GOLD): A double-blind, randomised, placebo-controlled, phase 3 trial. Lancet Oncol. 2017, 18, 1637-1651. [CrossRef]

11. Ross-Innes, C.S.; Wheatley, T.; Weaver, J.M.; Lynch, A.G.; Kingsbury, Z.; Ross, M.T.; Humphray, S.; Bentley, D.; Fitzgerald, R.C.; Becq, J.; et al. Whole-genome sequencing provides new insights into the clonal architecture of Barrett's esophagus and esophageal adenocarcinoma. Nat. Genet. 2015, 47, 1038-1046. [CrossRef] [PubMed]

12. Stachler, M.D.; Taylor-Weiner, A.; Peng, S.; McKenna, A.; Agoston, A.T.; Odze, R.D.; Davison, J.M.; Nason, K.S.; Loda, M.; Leshchiner, I.; et al. Paired exome analysis of Barrett's esophagus and adenocarcinoma. Nat. Genet. 2015, 47, 1047-1055. [CrossRef] [PubMed]

13. Dulak, A.M.; Stojanov, P.; Peng, S.; Lawrence, M.S.; Fox, C.; Stewart, C.; Bandla, S.; Imamura, Y.; Schumacher, S.E.; Shefler, E.; et al. Exome and whole-genome sequencing of esophageal adenocarcinoma identifies recurrent driver events and mutational complexity. Nat. Genet. 2013, 45, 478-486. [CrossRef] [PubMed]

14. Chong, I.Y.; Cunningham, D.; Barber, L.J.; Campbell, J.; Chen, L.; Kozarewa, I.; Fenwick, K.; Assiotis, I.; Guettler, S.; GarciaMurillas, I.; et al. The genomic landscape of oesophagogastric junctional adenocarcinoma. J. Pathol. 2013, 231, 301-310. [CrossRef] [PubMed]

15. The Cancer Genome Atlas Research Network Integrated genomic characterization of oesophageal carcinoma. Nat. Cell Biol. 2017, 541, 169-175. [CrossRef] 
16. Yakovleva, A.; Plieskatt, J.L.; Jensen, S.; Humeida, R.; Lang, J.; Li, G.; Bracci, P.; Silver, S.; Bethony, J.M. Fit for genomic and proteomic purposes: Sampling the fitness of nucleic acid and protein derivatives from formalin fixed paraffin embedded tissue. PLOS ONE 2017, 12, e0181756. [CrossRef]

17. Li, H.; Durbin, R. Fast and accurate short read alignment with Burrows-Wheeler transform. Bioinformatics 2009, 25, 1754-1760. [CrossRef]

18. McKenna, A.; Hanna, M.; Banks, E.; Sivachenko, A.; Cibulskis, K.; Kernytsky, A.; Garimella, K.; Altshuler, D.; Gabriel, S.B.; Daly, M.J.; et al. The Genome Analysis Toolkit: A MapReduce framework for analyzing next-generation DNA sequencing data. Genome Res. 2010, 20, 1297-1303. [CrossRef]

19. Greytak, S.R.; Engel, K.B.; Bass, B.P.; Moore, H.M. Accuracy of Molecular Data Generated with FFPE Biospecimens: Lessons from the Literature. Cancer Res. 2015, 75, 1541-1547. [CrossRef]

20. Futreal, P.A.; Coin, L.; Marshall, M.; Down, T.A.; Hubbard, T.; Wooster, R.; Rahman, N.; Stratton, M.R. A census of human cancer genes. Nat. Rev. Cancer 2004, 4, 177-183. [CrossRef]

21. Marusyk, A.; Almendro, V.; Polyak, K. Intra-tumour heterogeneity: A looking glass for cancer? Nat. Rev. Cancer 2012, 12, 323-334. [CrossRef] [PubMed]

22. Stahl, P.; Seeschaaf, C.; Lebok, P.; Kutup, A.; Bockhorn, M.; Izbicki, J.R.; Bokemeyer, C.; Simon, R.; Sauter, G.; Marx, A.H. Heterogeneity of amplification of HER2, EGFR, CCND1 and MYC in gastric cancer. BMC Gastroenterol. 2015, 15, 1-13. [CrossRef] [PubMed]

23. Wakatsuki, T.; Yamamoto, N.; Sano, T.; Chin, K.; Kawachi, H.; Takahari, D.; Ogura, M.; Ichimura, T.; Nakayama, I.; Osumi, H.; et al. Clinical impact of intratumoral HER2 heterogeneity on trastuzumab efficacy in patients with HER2-positive gastric cancer. J. Gastroenterol. 2018, 53, 1186-1195. [CrossRef] [PubMed]

24. Mroz, E.A.; Rocco, J.W. MATH, a novel measure of intratumor genetic heterogeneity, is high in poor-outcome classes of head and neck squamous cell carcinoma. Oral Oncol. 2013, 49, 211-215. [CrossRef] [PubMed]

25. Normanno, N.; Rachiglio, A.M.; Roma, C.; Fenizia, F.; Esposito, C.; Pasquale, R.; La Porta, M.L.; Iannaccone, A.; Micheli, F.; Santangelo, M.; et al. Molecular diagnostics and personalized medicine in oncology: Challenges and opportunities. J. Cell. Biochem. 2013, 114, 514-524. [CrossRef]

26. Esteve-Codina, A.; Arpí, O.; Martinez-García, M.; Pineda, E.; Mallo, M.; Gut, M.; Carrato, C.; Rovira, A.; López, R.; Tortosa, A.; et al. A Comparison of RNA-Seq Results from Paired Formalin-Fixed Paraffin-Embedded and Fresh-Frozen Glioblastoma Tissue Samples. PLoS ONE 2017, 12, e0170632. [CrossRef]

27. Suciu, B.A.; Pap, Z.; Dénes, L.; Brînzaniuc, K.; Copotoiu, C.; Pávai, Z. Allele-specific PCR method for identification of EGFR mutations in non-small cell lung cancer: Formalin-fixed paraffin-embedded tissue versus fresh tissue. Romanian J. Morphol. Embryol. Rev. Roum. Morphol. Embryol. 2016, 57, 495-500.

28. Lehmann, U.; Kreipe, H. Real-Time PCR Analysis of DNA and RNA Extracted from Formalin-Fixed and Paraffin-Embedded Biopsies. Methods 2001, 25, 409-418. [CrossRef]

29. Grünberg, J.; Verocay, M.C.; Rébori, A.; Pouso, J. Comparison of chronic peritoneal dialysis outcomes in children with and without spina bifida. Pediatr. Nephrol. 2007, 22, 573-577. [CrossRef]

30. De Paoli-Iseppi, R.; Johansson, P.A.; Menzies, A.M.; Dias, K.-R.; Pupo, G.M.; Kakavand, H.; Wilmott, J.S.; Mann, G.J.; Hayward, N.K.; Dinger, M.E.; et al. Comparison of whole-exome sequencing of matched fresh and formalin fixed paraffin embedded melanoma tumours: Implications for clinical decision making. Pathology 2016, 48, 261-266. [CrossRef]

31. Spencer, D.H.; Sehn, J.K.; Abel, H.J.; Watson, M.A.; Pfeifer, J.D.; Duncavage, E.J. Comparison of Clinical Targeted Next-Generation Sequence Data from Formalin-Fixed and Fresh-Frozen Tissue Specimens. J. Mol. Diagn. 2013, 15, 623-633. [CrossRef] [PubMed]

32. Solassol, J.; Ramos, J.; Lopez-Crapez, E.; Saifi, M.; Mangé, A.; Vianès, E.; Lamy, A.; Costes, V.; Maudelonde, T. KRAS Mutation Detection in Paired Frozen and Formalin-Fixed Paraffin-Embedded (FFPE) Colorectal Cancer Tissues. Int. J. Mol. Sci. 2011, 12, 3191-3204. [CrossRef] [PubMed]

33. Betge, J.; Kerr, G.; Miersch, T.; Leible, S.; Erdmann, G.; Galata, C.L.; Zhan, T.; Gaiser, T.; Post, S.; Ebert, M.P.; et al. Amplicon Sequencing of Colorectal Cancer: Variant Calling in Frozen and Formalin-Fixed Samples. PLoS ONE 2015, 10, e0127146. [CrossRef] [PubMed]

34. Gao, X.H.; Li, J.; Gong, H.F.; Yu, G.Y.; Liu, P.; Hao, L.Q.; Liu, L.J.; Bai, C.G.; Zhang, W. Comparison of Fresh Frozen Tissue with Formalin-Fixed Paraffin-Embedded Tissue for Mutation Analysis Using a Multi-Gene Panel in Patients With Colorectal Cancer. Front. Oncol. 2020, 10, 310. [CrossRef]

35. Do, H.; Dobrovic, A. Sequence Artifacts in DNA from Formalin-Fixed Tissues: Causes and Strategies for Minimization. Clin. Chem. 2015, 61, 64-71. [CrossRef] 